Tôhoku Math. Journ.

28 (1976), 277-292.

\title{
PICARD PRINCIPLE AND RIEMANN THEOREM
}

\author{
Mitsuru NAKaI
}

(Received March 20, 1975)

Consider the punctured unit Euclidean $m$-ball $B: 0<|x|<1\left(x=\left(x^{1}\right.\right.$, $\left.\left.\cdots, x^{m}\right), m \geqq 2\right)$ and a harmonic function $u(x)$ on $B$, i.e. $\Delta u=0(\Delta=$ $\left.\delta^{i j} \partial^{2} / \partial x^{i} \partial x^{j}\right)$ on $B$. The additional requirement that $u(x)$ has an upper or lower bound implies that $u(x)=c S(x)+h(x)$ where $c$ is a constant, $h(x)$ a harmonic function on $|x|<1$, and $S(x)$ the fundamental singularity $|x|^{2-m}(m \geqq 3)$ or $-\log |x|(m=2)$. This classical result, the so called principle of positive singularity, originally obtained by Picard for $m=2$, is reformulated by Bouligand under the name Picard principle as follows: The dimension of the half module of nonnegative harmonic functions on $B$ with vanishing boundary values zero on $|x|=1$ is one. As a result the Riemann theorem on removable singularities follows, the weak form of which states that the boundedness of $u(x)$ on $B$ yields the existence of $\lim _{x \rightarrow 0} u(x)$. Conversely the Picard principle can be derived from the Riemann theorem as the original proof of Picard on Picard principle suggests, and therefore the Picard principle and the Riemann theorem are equivalent in essence. The primary purpose of this paper is to discuss this duality relation between the Picard principle and the Riemann theorem if the base region $B$ and the operator $\Delta$ are generalized to $C^{\infty}$-manifolds $\Omega$ and second order elliptic differential operators $L$.

Consider an $m$-dimensional separable connected orientable $C^{\infty}$-manifold $M(m \geqq 2)$. A regular subregion $N$ of $M$ is a relatively compact subregion whose relative boundary $\partial N$ consists of a finite number of disjoint $(m-1)$ dimensional closed simple hypersurface of class $C^{3}$. If in addition $M-N$ has no compact component, then $N$ will be referred to as a normal subregion. We always assume that $M$ has a single ideal boundary component $\delta$, i.e. the directed net $\mathscr{N}(M)=\{N\}$ of normal subregions $N$ of $M$ is nonvoid and $M-\bar{N}$ is connected for every $N$ in $\mathscr{N}(M)$. In this case $\Omega=M-\bar{N}(N \in \mathscr{N}(M))$ is called an end of $M$. Then the directed net $\omega(M)=\{\Omega\}$ of ends of $M$ forms a base of punctured neighborhood of $\delta$. Clearly $B$ can be viewed as an end of a suitable admitted manifold. Consider an elliptic differential operator $L$ on an end $\Omega$ defined by

$$
L u(x)=\frac{1}{\sqrt{a(x)}} \frac{\partial}{\partial x^{i}}\left(\sqrt{a(x)} a^{i j}(x) \frac{\partial}{\partial x^{j}} u(x)\right)+b^{i}(x) \frac{\partial}{\partial x^{i}} u(x)+c(x) u(x)
$$


for $u \in C^{2}(\Omega)$ where $\left(a^{i j}(x)\right)$ and $\left(b^{i}(x)\right)$ are contravariant tensors of class $C^{2}$ on $\Omega, c(x)$ is a function of class $C^{1}$ on $\Omega,\left(a^{i j}(x)\right)$ is symmetric and strictly positive definite for each $x \in \Omega$, and $a(x)=\operatorname{det}\left(a^{i j}(x)\right)^{-1}$. After Bouligand we say that the Picard principle is valid for $L$ if the dimension of the half module of nonnegative solutions of (1) on $\Omega$ with vanishing boundary values on $\partial \Omega$ is one. We also say that the Riemann theorem is valid for $L$ if $\lim _{x \rightarrow \delta} u(x)$ exists for every bounded solution of (1) on $\Omega$ with continuous boundary values on $\partial \Omega$.

In the case when $L$ is defined on $\bar{\Omega}=\Omega \cup \partial \Omega$ and $\Omega$ tolerates the Green's function $G_{\Omega}(\cdot, y)$ with pole at any point $y$ in $\Omega$ with respect to $L$, we associate an elliptic differential operator $\hat{L}$ with $L$ defined by

$$
\begin{gathered}
\hat{L} u(x)=\frac{1}{\sqrt{a(x)}} \frac{\partial}{\partial x^{i}}\left(\sqrt{a(x)} a^{i j}(x) \frac{\partial}{\partial x^{j}} u(x)\right)-b^{i}(x) \frac{\partial}{\partial x^{i}} u(x) \\
+2 a^{i j}(x) \frac{\partial}{\partial x^{i}} \log e_{\Omega}(x) \frac{\partial}{\partial x^{j}} u(x)
\end{gathered}
$$

for $u \in C^{2}(\Omega)$. Here the function $e_{\Omega}(x)$ is given by

$$
e_{\Omega}(y)=\int_{\partial 2} \frac{\partial}{\partial n_{x}} G_{\Omega}(x, y) d S_{x}
$$

where $\partial / \partial n$ denotes the inner normal derivative and $d S$ the surface element with respect to the Riemannian metric defined by the tensor $\left(a_{i j}(x)\right)=$ $\left(a^{i j}(x)\right)^{-1}$. The primary purpose of this paper is to prove the following duality relation:

TheOREM. The Picard principle is valid for an operator $L$ if and only if the Riemann theorem is valid for the associated operator $\hat{L}$.

This was originally obtained by Heins [3] for the case when $\Omega$ is an end of a parabolic Riemann surface $M$ and $L=\Delta$. Hayashi [2] removed the parabolicity assumption on the Heins result and generalized $\Delta$ to $\Delta-P(P \geqq 0)$. If $\Omega=B$ and $L=\Delta$, then $\hat{L}=\Delta$ and the above theorem assures the equivalence of the classical Picard principle and the classical Riemann theorem. In this connection we add here the following remark on the Picard principle. Consider an operator $L_{\lambda}$ on the punctured unit disk $0<|z|<1$ on the complex plane defined by

$$
L_{\lambda} u(z)=\Delta u(z)-|z|^{-\lambda} u(z)
$$

where we take $z=0$ to be $\delta$. Then the Picard principle is valid for $L_{\lambda}$ if and only if $\lambda \in[-\infty, 2]$ (Nakai [6]). In view of this one might get the feeling that in order the Picard principle to hold for an operator $L u=\Delta u+b \cdot \nabla u+c u$ on $0<|z|<1$ at $z=0$ the singularities of $b$ and 
$c$ at $z=0$ must be 'naive'. However this is not the case. For example consider the operator

$$
L_{P} u(z)=\Delta u(z)+\nabla\left(2 \log e_{P}(z)\right) \cdot \nabla u(z)+P(z) u(z)
$$

where $P(z)$ is an arbitrary nonnegative $C^{1}$-function on $0<|z| \leqq 1$ and $e_{P}(z)$ is the unique bounded solution of $\Delta u(z)=P(z) u(z)$ on $0<|z|<1$ with the boundary values 1 on $|z|=1$. We shall see as an application of the above that the Picard principle is valid for $L_{P}$ for any $P \geqq 0$ which may behave as wildly as we wish at $z=0$.

\section{Elliptic dimensions.}

1. With respect to the Riemannian metric $d s^{2}=a_{i j}(x) d x^{i} d x^{j}$ on an end $\tilde{\widetilde{\Omega}}$ the Laplace-Beltrami operator $\Delta$ takes the form

$$
\Delta u(x)=\frac{1}{\sqrt{a(x)}} \frac{\partial}{\partial x^{i}}\left(\sqrt{a(x)} a^{i j}(x) \frac{\partial}{\partial x^{j}} u(x)\right)
$$

for $u \in C^{2}(\Omega)$. Let $b(x)=\left(b_{1}(x), \cdots, b_{m}(x)\right)$ and $\bar{b}(x)=\left(\bar{b}_{1}\left(x_{1}\right), \cdots, \bar{b}_{m}(x)\right)$ be covariant tensors on $\Omega$. The inner product $b \cdot \bar{b}$ with respect to the metric $d s$ is given by

$$
b(x) \cdot \bar{b}(x)=a^{i j}(x) b_{i}(x) \bar{b}_{j}(x) .
$$

Using the contravariant tensor $\left(b^{i}(x)\right)$ in (1) we form the covariant tensor

$$
b(x)=\left(b^{1}(x), \cdots, b^{m}(x)\right)\left(a_{i j}(x)\right) .
$$

We denote by $\nabla u(x)$ be covariant tensor $\left(\partial u(x) / \partial x^{1}, \cdots, \partial u(x) / \partial x^{m}\right)$. With the aid of these notations (1) can be represented as

$$
L u(x)=\Delta u(x)+b(x) \cdot \nabla u(x)+c(x) u(x) .
$$

The adjoint operator $L^{*}$ of $L$ then takes the form

$$
L^{*} u(x)=\Delta u(x)-b(x) \cdot \nabla u(x)+c^{*}(x) u(x)
$$

where the function $c^{*}$ is given by

$$
c^{*}(x)=c(x)-\frac{1}{\sqrt{a(x)}} \frac{\partial}{\partial x^{i}}\left(\sqrt{a(x)} a^{i j}(x) b_{j}(x)\right) .
$$

In case $L$ is defined on $\bar{\Omega}$, which we shall assume throughout this paper unless the contrary is stated, and $\Omega$ torelates the Green's function for $L$, the function $e_{\Omega}(x)$ in (3) is a positive solution of $L^{*} u=0$ on $\Omega$ with boundary values 1 on $\partial \Omega$, and the associated operator $\hat{L}$ to $L$ is represented as

$$
\hat{L} u(x)=\Delta u(x)+\left(2 \nabla \log e_{i \dot{\alpha}}(x)-b(x)\right) \cdot \nabla u(x)
$$


2. We denote by $\mathscr{P}(\Omega ; L)$ the half module of nonnegative solutions of (4) with vanishing boundary values zero on $\partial \Omega$. We also denote by $\mathscr{E}(\Omega ; L)$ the linear space generated by $\mathscr{P}(\Omega ; L)$, i.e. $\mathscr{E}(\Omega ; L)=\mathscr{P}(\Omega ; L) \Theta$ $\mathscr{P}(\Omega ; L)=\left\{u_{1}-u_{2} ; u_{i} \in \mathscr{P}(\Omega ; L)(i=1,2)\right\}$. Then $\mathscr{E}(\Omega ; L)$ is a metrizable locally convex linear space with the topology induced by convergence on every compact subset of $\Omega$. Let

$$
\sigma(u)=\int_{\partial \Omega} \frac{\partial}{\partial n_{x}} u(x) d S_{x}
$$

for $u \in \mathscr{E}(\Omega ; L)$ which defines a continuous linear functional on $\mathscr{E}(\Omega ; L)$, i.e. $\sigma \in \mathscr{E}(\Omega ; L)^{*}$. Consider the set

$$
\mathscr{P}_{1}(\Omega ; L)=\{u \in \mathscr{P}(\Omega ; L) ; \sigma(u)=1\},
$$

which is convex and also compact in $\mathscr{E}(\Omega ; L)$ by the Harnack principle. Observe that $\mathscr{P}_{1}(\Omega ; L)$ is contained in the closed hyperplane $\{u \in \mathscr{E}(\Omega ; L)$; $\sigma(u)=1\}$ which misses the origin. Since $\sigma(u)=0$ for $u \in \mathscr{P}(\Omega ; L)$ implies that

$$
\frac{\partial}{\partial n_{x}} u(x)=u(x)=0
$$

on $\partial \Omega, \sigma(u)=0$ is equivalent to $u=0$ on $\Omega$ (cf. e.g. Miranda [5]). Therefore $\mathscr{P}_{1}(\Omega ; L)$ is the base of the cone $\mathscr{P}(\Omega ; L)$, i.e. for any $v \in \mathscr{P}(\Omega ; L)$ there exist a unique $u \in \mathscr{P}_{1}(\Omega ; L)$ and a unique nonnegative number $\lambda$ with $v=\lambda u$. The cone $\mathscr{P}(\Omega ; L)$ induces an order $u \geqq v$ on $\mathscr{E}(\Omega ; L)$ by $u-v \in \mathscr{P}(\Omega ; L)$, which is the usual pointwise function ordering $u(x) \geqq$ $v(x)$ on $\Omega$. We maintain that $\mathscr{P}_{1}(\Omega ; L)$ is a simplex, i.e. $\mathscr{E}(\Omega ; L)$ is a vector lattice, which is equivalent to that $\mathscr{P}(\Omega ; L)$ is a lattice. This is clear if $\mathscr{P}(\Omega ; L)=\{0\}$ and we suppose that $\mathscr{P}(\Omega ; L)$ contains an $h>0$. Let $u$ and $v$ be in $\mathscr{P}(\Omega ; L)$. For each $N \in \mathscr{N}(M)$ with $N \supset M-\Omega$, there exists a unique solution $w_{N}$ of (4) on $N \cap \Omega$ with boundary values zero on $\partial \Omega$ and $\max (u(x), v(x))$ on $\partial N$. The unique existence of $w_{N}$ follows from the existence of $h$. Observe that

$$
u, v \leqq w_{N} \leqq u+v
$$

on $N \cap \Omega$. Therefore $w_{N} \leqq w_{N^{\prime}}$ if $N \subset N^{\prime}$ and $w=\lim _{N \rightarrow M} w_{N}$ exists on $\Omega$ which belongs to $\mathscr{P}(\Omega ; L)$. If an $s \in \mathscr{P}(\Omega ; L)$ dominates both $u$ and $v$, then $w_{N} \leqq s$ on $N \cap \Omega$ and a fortiori $w \leqq s$ on $\Omega$. This means that $w$ is the least upper bound of $u$ and $v$. Similarly we can see the existence of the greatest lower bound of $u$ and $v$ and thus $\mathscr{P}(\Omega ; L)$ is a lattice.

3. We denote by Ex. $\mathscr{P}_{1}(\Omega ; L)$ the set of extreme points of $\mathscr{P}_{1}(\Omega ; L)$. Since $\mathscr{P}_{1}(\Omega ; L)$ is a compact convex subset of $\mathscr{E}(\Omega ; L)$ and is also a 
simplex, the Choquet theorem (cf. e.g. Phelps [8]) assures that there exists a bijective correspondence $u \leftrightarrow \nu$ between $\mathscr{P}(\Omega ; L)$ and the set of regular Borel measures on Ex. $\mathscr{P}_{1}(\Omega ; L)$ such that

$$
u=\int_{\mathrm{Ex} \cdot \mathscr{V}_{1}(\Omega ; L)} v d \nu(v) \text {. }
$$

We define the elliptic dimension of $(\Omega ; L), \operatorname{dim}(\Omega ; L)$ in notation, by the dimension of the half module $\mathscr{P}(\Omega ; L)$. In view of (9) we thus define

$$
\operatorname{dim}(\Omega ; L)=\#\left(\operatorname{Ex} . \mathscr{P}_{1}(\Omega ; L)\right)
$$

where \# denotes the cardinal number. We say that the Picard principle is valid for $(\Omega ; L)$ if $\operatorname{dim}(\Omega ; L)=1$.

\section{Principal functions.}

4. In nos. 4-5 we assume that the operator $L$ in (4) is defined only on an end $\Omega_{0}$ of $M$, not necessarily on $\bar{\Omega}_{0}$, and that there exists a strictly positive solution $h$ of (4) on $\Omega_{0}$ with vanishing boundary values on $\partial \Omega_{0}$. Observe that $v$ is a solution of (4) on an open subset of $\Omega_{0}$ if and only if $u=v / h$ is a solution of

$$
L_{h} u(x)=\Delta u(x)+(2 \nabla \log h(x)+b(x)) \cdot \nabla u(x) .
$$

Since the Dirichlet problem for (11) is uniquely solvable for any normal subregion of $\Omega_{0}$, the same is true for the operator (4). Let $N \in \mathscr{N}(M)$ with $N \supset M-\Omega_{0}$. Even if $L$ is defined on $\bar{\Omega}_{0}, L_{h}$ need not be defined on $\bar{\Omega}_{0}$. Therefore the solvability of the Dirichlet problem for $L$ on $N \cap \Omega_{0}$ may not be assured.

Let $\Omega$ be an end of $M$ with $\bar{\Omega} \subset \Omega_{0}$. Take $N \in \mathscr{N}(M)$ so large that $N \supset M-\Omega$. Consider a solution $B_{N} \varphi$ of (11) on $N \cap \Omega$ with boundary values zero on $\partial N$ and $\phi \in C(\partial \Omega)$ on $\partial \Omega$. Set $k=\max _{\partial \Omega}|\varphi|$. Observe that $\left|B_{N} \varphi\right| \leqq k$ on $N \cap \Omega$. If $\varphi \geqq 0$, then $B_{N} \varphi \leqq B_{N^{\prime}}, \varphi\left(\bar{N} \subset N^{\prime}\right)$. Therefore

$$
B \varphi=\lim _{N \rightarrow M} B_{N} \varphi
$$

exists on $\Omega$. The same is true for general $\varphi$ by considering $\varphi=\varphi^{+}-\varphi^{-}$ with $\phi^{ \pm}=\max ( \pm \varphi, 0)$. Then $B=B(\Omega ; \partial \Omega)$ defines a positive linear operator from $C(\partial \Omega)$ into $C(\bar{\Omega})$ such that $B \varphi$ is a solution of (11) on $\Omega$ and

$$
B \varphi|\partial \Omega=\varphi, \quad| B \varphi\left|\leqq \max _{\partial \Omega}\right| \varphi \mid \cdot
$$

Set $D_{N} \varphi=h B_{N}(\varphi / h)$ which is a solution of (4) on $N \cap \Omega$ with boundary values zero on $\partial N$ and $\varphi$ on $\partial \Omega$. As a counter part of (12) we have

$$
D \varphi=\lim _{N \rightarrow M} D_{N} \varphi
$$


on $\Omega$. Then $D=D(\Omega ; \partial \Omega)$ defines a positive linear operator from $C(\partial \Omega)$ into $C(\bar{\Omega})$ such that $D \varphi$ is a solution of (4) on $\Omega$ and, as a counterpart of (13),

$$
D \varphi|\partial \Omega=\varphi, \quad| D \varphi \mid \leqq\left(\max _{\partial \Omega}|\varphi / h|\right) h .
$$

Next take an $N_{0} \in \mathscr{N}(M)$ such that $N_{0} \supset\left(M-\Omega_{0}\right) \cup(\partial \Omega)$. Let $N \in$ $\mathscr{N}(M)$ with $M-\Omega_{0} \subset N \subset \bar{N} \subset N_{0}$ and $B_{N} \varphi$ be the solution of (11) on $N_{0}-\bar{N}$ with boundary values $\varphi \in C\left(\partial N_{0}\right)$ an $\partial N_{0}$ and zero on $\partial N$. Similarly as (12) we have the existence of

$$
B \varphi=\lim _{N \rightarrow M-\Omega_{0}} B_{N} \varphi
$$

on $N_{0} \cap \Omega_{0}$. Then $B=B\left(N_{0} \cap \Omega_{0} ; \partial N_{0}\right)$ is a positive linear operator from $C\left(\partial N_{0}\right)$ into $C\left(\overline{N_{0} \cap \Omega_{0}}\right)$ such that $B \varphi$ is a solution of (11) on $\Omega$ and

$$
B \varphi\left|\partial N_{0}=\varphi, \quad\right| B \varphi\left|\leqq \max _{\partial N_{0}}\right| \varphi \mid \text {. }
$$

Set $D \varphi=h B(\varphi / h)$. Then $D=D\left(N_{0} \cap \Omega_{0} ; \partial N_{0}\right)$ is a positive linear operator from $C\left(\partial N_{0}\right)$ into $C\left(\bar{N}_{0} \cap \Omega_{0}\right)$ such that $D \varphi$ is a solution of (4) in $N_{0} \cap \Omega_{0}$ and, as a consequence of (17),

$$
D \varphi\left|\partial \Omega_{0}=0, \quad D \varphi\right| \partial N_{0}=\varphi .
$$

5. Suppose $s \in C\left(\bar{N}_{0} \cap \Omega_{0}\right)$ is a solution of (4) on $N_{0} \cap \Omega_{0}$. According to Sario (cf. e.g. Rodin-Sario [9]) we say that a function $p$ on $\Omega_{0}$ is a principal function relative to $\left(s, D\left(N_{0} \cap \Omega_{0} ; \partial N_{0}\right), D(\Omega ; \partial \Omega)\right)$ if

$$
D\left(N_{0} \cap \Omega_{0} ; N_{0}\right)(p-s)=p-s, \quad D(\Omega ; \partial \Omega) p=p .
$$

On setting $\sigma=s / h, p$ is a solution to (19) if and only if $q=p / h$ is a solution to

$$
B\left(N_{0} \cap \Omega_{0} ; \partial N_{0}\right)(q-\sigma)=q-\sigma, \quad B(\Omega ; \partial \Omega) q=q .
$$

We shall show that (20) has a unique solution. For this purpose we consider an operator $T: C(\partial \Omega) \rightarrow C(\partial \Omega)$ defined by

$$
T \varphi=B\left(N_{0} \cap \Omega_{0} ; \partial N_{0}\right)(B(\Omega ; \partial \Omega) \varphi) .
$$

In view of (13), (17), and the Harnack principle, we see that $T$ is a compact operator. Again by (13), (17), and the maximum principle, we deduce that $T \varphi=\varphi$ implies $\phi=0$, i.e. 1 is not the proper value of $T$. Therefore the Riesz-Schauder theory (cf. Yosida [11]) assures the existence of $\varphi \in C(\partial \Omega)$ with

$$
(I-T) \varphi=\sigma_{0}
$$

where $\sigma_{0}=\sigma-B\left(N_{0} \cap \Omega_{0} ; \partial N_{0}\right) \sigma$. We define $q$ on $\Omega_{0}$ by 


$$
q(x)=\left\{\begin{array}{lr}
(B(\Omega ; \partial \Omega) \varphi)(x) & (x \in \Omega) ; \\
\left(B\left(N_{0} \cap \Omega_{0} ; \partial N_{0}\right)(B(\Omega ; \partial \Omega) \varphi)\right)(x)+\sigma_{0}(x) & \left(x \in N_{0} \cap \Omega_{0}\right) .
\end{array}\right.
$$

It is easy to see that $q$ is well defined on $\Omega_{0}$ and satisfies (20). The uniqueness of $q$ follows from (13), (17), and the maximum principle.

Let $N \in \mathscr{N}(M)$ be so large that $N \supset N_{0}$. By exactly the same method as above we see that there exists a unique solution $p$ on $N \cap \Omega_{0}$ to

$$
D\left(N_{0} \cap \Omega_{0} ; \partial N_{0}\right)(p-s)=p-s, \quad D(N \cap \Omega ; \partial \Omega) p=p,
$$

and $q=p / h$ is a unique solution on $N \cap \Omega_{0}$ to

$$
B\left(N_{0} \cap \Omega_{0} ; \partial N_{0}\right)(q-\sigma)=q-\sigma, \quad B(N \cap \Omega ; \partial \Omega) q=q .
$$

6. We now assume that $L$ is defined on $\bar{\Omega}_{0}$. We assert that the existence of a solution $u_{N}$ of (4) on $N \cap \Omega_{0}$ with boundary values $\varphi \in$ $C\left(\partial \Omega_{0}\right)$ on $\partial \Omega_{0}$ and zero on $\partial N$ for every $N \in \mathscr{N}(M)$ with $N \supset M-\Omega_{0}$. Let $N_{0} \in \mathscr{N}(M)$ with $M-\Omega_{0} \subset N_{0} \subset \bar{N}_{0} \subset N$. If the measure of $N_{0} \cap \Omega_{0}$ is sufficiently small, then there exists a unique solution $s$ of (4) on $N_{0} \cap \Omega_{0}$ with boundary values $\varphi$ on $\partial \Omega_{0}$ and zero on $\partial N_{0}$ (cf. e.g. [5]). Then the principal function satisfying (21) is the required $u$. We denote by $D\left(N \cap \Omega_{0}, \partial \Omega_{0}\right) \varphi$ the so constructed $u_{N}$. We also denote by $u$ the principal function on $\Omega_{0}$ satisfying (19). Suppose $\varphi \geqq 0$ and thus $s \geqq 0$. We maintain that $u \geqq 0$ and $u_{N} \geqq 0$, or equivalently $v=u / h \geqq 0$ and $v_{N}=u_{N} / h \geqq 0$. Suppose $\min _{\partial N_{0}} v<0$. Then

$$
\min _{\bar{N}_{0} \cap \Omega_{0}} v=\min _{\bar{N}_{0} \cap \Omega_{0}}\left(\sigma+B\left(N_{0} \cap \Omega ; \partial N_{0}\right)(v-\sigma)\right) \geqq \min _{\partial N_{0}} v .
$$

Therefore $\min _{\partial \Omega} v<0$ and $\min _{\bar{\Omega}} v=\min _{\partial \Omega} v \geqq \min _{\partial N_{0}} v$. These mean that $v$ considered on $\Omega_{0}$ takes its minimum on $\partial N_{0}$ which contradicts the minimum principle. Thus $v \geqq 0$ on $\Omega_{0}$ and similarly $v_{N} \geqq 0$ on $N \cap \Omega_{0}$. A fortiori $u_{N} \leqq u_{N^{\prime}} \leqq u$ for $\bar{N} \subset N^{\prime}$ and we conclude the existence of

$$
D \varphi=\lim _{N \rightarrow M} D\left(N \cap \Omega_{0} ; \partial \Omega_{0}\right) \varphi
$$

on $\Omega_{0}$. This is also true for general $\varphi$ since we only have to consider $\phi=\varphi^{+}-\phi^{-}$. Therefore $D=D\left(\Omega_{0} ; \partial \Omega_{0}\right)$ is a positive linear operator from $C\left(\partial \Omega_{0}\right)$ into $C\left(\bar{\Omega}_{0}\right)$ such that $D \varphi$ is a solution of (4) on $\Omega_{0}$ and

$$
D \varphi \mid \partial \Omega_{0}=\varphi \text {. }
$$

The operator $D\left(\Omega_{0} ; \partial \Omega_{0}\right)$ will play an important role in our discussions.

7. We shall prove that the Picard principle as well as the elliptic dimension is the property of ideal boundary $\delta$. Let $\Omega_{0}$ be an end of $M$ such that $L$ is defined on $\bar{\Omega}_{0}$. We maintain (cf. Ozawa [7]): 
Proposition. If $\operatorname{dim}\left(\Omega_{0} ; L\right)>0$, then $\operatorname{dim}(\Omega ; L)=\operatorname{dim}\left(\Omega_{0} ; L\right)$ for every end $\Omega$ of $M$ with $\bar{\Omega} \subset \Omega_{0}$.

Since $\operatorname{dim}\left(\Omega_{0} ; L\right)>0$, we fix an $h \in \mathscr{P}\left(\Omega_{0} ; L\right)$ with $h>0$ on $\Omega_{0}$. Consider a mapping $\pi: \mathscr{P}\left(\Omega_{0} ; L\right) \rightarrow \mathscr{P}(\Omega ; L)$ defined by $\pi u=u-D(\Omega ; \partial \Omega) u$. To see that $\pi$ is injective, let $\pi u_{1}=\pi u_{2}$ for $u_{j} \in \mathscr{P}\left(\Omega_{0} ; L\right)(j=1,2)$. On setting $v=\left(u_{1}-u_{2}\right) / h$, we see that $B(\Omega ; \partial \Omega) v=v$ and clearly $B\left(\Omega_{0}-\bar{\Omega}\right.$; $\partial \Omega) v=v$. Therefore $v$ considered on $\Omega_{0}$ takes its maximum on $\partial \Omega$ and a fortiori $v$ is a constant. Thus

$$
D\left(N_{0} \cap \Omega_{0} ; \partial N_{0}\right) h=h, \quad D(\Omega ; \partial \Omega) h=h,
$$

where $N_{0} \in \mathscr{N}(M)$ with $M-\Omega \subset N_{0} \subset \bar{N}_{0} \subset M-\bar{\Omega}$. This means that $h$ is a principal function with respect to $\left(0, D\left(N_{0} \cap \Omega_{0} ; \partial N_{0}\right), D(\Omega ; \partial \Omega)\right)$, and by the uniqueness of a principal function, $h=0$, which is a contradiction. To prove that $\pi$ is surjective, let $s \in \mathscr{P}(\Omega ; L)$. We wish to show the existence of a $p$ on $\Omega_{0}$ with

$$
D\left(N_{0} \cap \Omega_{0} ; \partial N_{0}\right) p=p, \quad D(\Omega ; \partial \Omega)(p-s)=p-s,
$$

where this time we take $N_{0} \in \mathscr{N}(M)$ so large that $M-\Omega \subset N_{0}$. Then we can conclude that $p \in \mathscr{P}\left(\Omega_{0} ; L\right)$ as in (6) and $\pi p=s$. The principal function problem (25) is equivalent to find $q=p / h$ with

$$
B\left(N_{0} \cap \Omega_{0} ; \partial N_{0}\right) q=q, \quad B(\Omega ; \partial \Omega)(q-\sigma)=q-\sigma
$$

with $\sigma=s / h$. As in 5, (26) is equivalent to finding $\varphi \in C\left(\partial N_{0}\right)$ such that

$$
(I-T) \varphi=\sigma
$$

where $\left.T_{\varphi}=B(\Omega ; \partial \Omega)\left(B\left(N_{0} \cap \Omega_{0}\right) ; \partial N_{0}\right) \varphi\right)$. By the same method as in 5 the above Fredholm equation is solved.

\section{Green's functions and Martin kernel.}

8. Fix an end $\Omega$ of $M$ and take an $N \in \mathscr{N}(M)$ with $M-\Omega \subset N$. The Green's function $G_{N \cap \Omega}(x, y)$ on $N \cap \Omega$ for $L$ with pole $y \in N \cap \Omega$ is a solution of $L$ on $N \cap G-\{y\}$, i.e. $L_{x} G_{N \cap G}(x, y)=0$, with vanishing boundary values on $\partial(N \cap \Omega)$ such that the unique solution $u$ of the Dirichlet problem for $L u=f, f$ being Hölder continuous on $\overline{N \cap \Omega}$, with $u \mid \partial(N \cap \Omega)=$ $\phi \in C(\partial(N \cap \Omega))$ is given by

$$
\begin{aligned}
u(x)= & -\int_{N \cap \Omega} G_{N \cap \Omega}(x, y) f(y) \sqrt{a(y)} d y^{1} \cdots d y^{m} \\
& +\int_{\partial(N \cap \Omega)} \frac{\partial G_{N \cap \Omega}(x, y)}{\partial n_{y}} \varphi(y) d S_{y}
\end{aligned}
$$

(cf. e.g. Miranda [5]). The function $G_{N \cap \Omega}(x, \cdot)$ is also the Green's function 
on $N \cap \Omega$ for $L^{*}$ with pole $x \in N \cap \Omega$. If $\operatorname{dim}(\Omega ; L)>0$, then the Green's function $G_{N \cap \Omega}(x, y)$ exists for any $N$ and

$$
D(N \cap \Omega ; \partial \Omega) \varphi=\int_{\partial \Omega} \frac{\partial G_{N \cap \Omega}(x, y)}{\partial n_{y}} \varphi(y) d S_{y},
$$

for $\varphi \in C(\partial \Omega)$. If $G_{N \cap \Omega}(x, y)$ exists for every $N$, then $\left\{G_{N \cap \Omega}(x, y)\right\}_{N}$ is an increasing net. In case it is convergent we call

$$
G_{\Omega}(x, y)=\lim _{N \rightarrow M} G_{N \cap s_{s}}(x, y)
$$

the Green's function on $\Omega$ for $L$ with pole $y \in \Omega$. If $\operatorname{dim}(\Omega ; L)>0$, then (23) and (28) imply the existence of (29) and

$$
D(\Omega ; \partial \Omega) \varphi=\int_{\partial \Omega} \frac{\partial G_{\Omega}(\cdot, y)}{\partial n_{y}} \varphi(y) d S_{y}
$$

for $\varphi \in C(\partial \Omega)$. Actually the converse is also true:

Proposition. The Green's function $G_{\Omega}(x, y)$ exists on $\Omega$ for $L$ if and only if $\operatorname{dim}(\Omega ; L)>0$, which is equivalent to $\operatorname{dim}\left(\Omega ; L^{*}\right)>0$.

Suppose $\operatorname{dim}(\Omega ; L)>0$ and let $h \in \mathscr{P}(\Omega ; L)$ with $h>0$ on $\Omega$. For an arbitrary point $y \in N \cap \Omega$ we take punctured parametric balls $U: 0<$ $|x-y|<a$ and $V: 0<|x-y|<b(0<a<b)$ such that $\bar{V} \subset N \cap \Omega$. If $V$ is sufficiently small, then $s(x)=G_{V}(x, y)$, the Green's function on $V$ for $L$, exists. The principal function $p$ on $N \cap \Omega-\{y\}$ with

$$
D(V ; \partial \bar{V})(p-s)=p-s, \quad D(N \cap \Omega-\bar{U} ; \partial U) p=p
$$

is seen to exists as in 5, and clearly $p(x)$ is the required $G_{N \cap \Omega}(x, y)$. From this as above the existence of $G_{\Omega}(x, y)$ follows. Conversely suppose the existence of $G_{\Omega}(x, y)$ on $\Omega$ for $L$, fix a point $x_{0} \in \Omega$ and consider $u_{y}(x)=$ $G_{\Omega}(x, y) / G_{\Omega}\left(x_{0}, y\right)$. Since $u_{y} \geqq 0$ and $u_{y}\left(x_{0}\right)=1$, the Harnack principle assures that $\left\{u_{y}(x) ; y \rightarrow \delta\right\}$ forms a normal family and thus we can find a sequence $\left\{y_{n}\right\}$ converging to $\delta$ such that $u(x)=\lim _{n \rightarrow \infty} u_{y_{n}}(x)$ exists on $\Omega$. Then $u>0$ and $u \in \mathscr{P}(\Omega ; L)$, i.e. $\operatorname{dim}(\Omega ; L)>0$. Similarly $G_{\Omega}(x, y)$ exists if and only if $\operatorname{dim}\left(\Omega ; L^{*}\right)>0$. A fortiori $\operatorname{dim}(\Omega ; L)>0$ is equivalent to $\operatorname{dim}\left(\Omega ; L^{*}\right)>0$.

9. If $\operatorname{dim}(\Omega ; L)>0$, then $\operatorname{dim}\left(\Omega ; L^{*}\right)>0$, and as in 6 we can define $D^{*}(\Omega \cap N ; \partial \Omega)$ and $D^{*}(\Omega ; \partial \Omega)$, i.e. $D^{*}(\Omega \cap N ; \partial \Omega) \varphi$ is a solution of $L^{*} u=0$ on $\Omega \cap N$ with boundary values $\varphi \in C(\partial \Omega)$ on $\partial \Omega$ and zero on $\partial N$, and

$$
D^{*}(\Omega ; \partial \Omega) \varphi=\lim _{N \rightarrow M} D^{*}(\Omega \cap N ; \partial \Omega) \varphi
$$

exists on $\Omega$. Then 


$$
D^{*}(\Omega \cap N ; \partial \Omega) \varphi=\int_{\partial \Omega} \frac{\partial G_{N \cap \cap}(x, \cdot)}{\partial n_{x}} \varphi(x) d S_{x}
$$

and similarly as in (30) we deduce

$$
D^{*}(\Omega ; \partial \Omega) \varphi=\int_{\partial \Omega} \frac{\partial G_{\Omega}(x, \cdot)}{\partial n_{x}} \varphi(x) d S_{x} .
$$

10. We assume that $\operatorname{dim}(\Omega ; L)>0$ or equivalently there exists the Green's function $G_{\Omega}(x, y)$ on $\Omega$ for $L$. The function

$$
e_{\Omega}(y)=\left(D^{*}(\Omega ; \partial \Omega) 1\right)(y)=\int_{\partial \Omega} \frac{\partial G_{\Omega}(x, y)}{\partial n_{x}} d S_{x}
$$

is a positive solution of $L^{*} u=0$ on $\Omega$ with boundary values 1 on $\partial \Omega$. Using the function $e_{\Omega}$ we define the Martin kernel $K_{s}(x, y)$ on $\Omega$ by

$$
K_{\delta}(x, y)=G_{\delta c}(x, y) / e_{\delta c}(y)
$$

for $(x, y) \in \bar{\Omega} \times \Omega$ with $x \neq y$ and $K_{\Omega}(x, x)=+\infty$ for $x \in \Omega$. Define a metric $\rho\left(y_{1}, y_{2}\right)$ on $\Omega$ given by

$$
\rho\left(y_{1}, y_{2}\right)=\sum_{n=1}^{\infty} \varepsilon_{n}\left|K_{\Omega}\left(x_{n}, y_{1}\right)-K_{\Omega}\left(x_{n}, y_{2}\right)\right|^{*}
$$

where $\varepsilon_{n}>0$ with $\sum_{n=1}^{\infty} \varepsilon_{n}=1,\left\{x_{n}\right\}_{n \geqq 1}$ a countable dense subset of $\Omega$, and $|\alpha|^{*}=|\alpha|(1+|\alpha|)^{-1}$ for real numbers $\alpha$ and $|+\infty|^{*}=1$. The metric space $(\Omega, \rho)$ is homeomorphic to the original topological space $\Omega$. Let $\Omega^{\prime}$ be an end of $M$ with $\bar{\Omega}^{\prime} \subset \Omega$. By (29) and (31), $\inf _{y \in \Omega} K(x, y)>0$ for every $x \in \Omega-\bar{\Omega}^{\prime}$. In view of this, $\rho$-Cauchy sequences $\left\{y_{n}\right\}$ in $\Omega$ are divided into three categories: $\left\{y_{n}\right\} \rightarrow \partial \Omega,\left\{y_{n}\right\} \rightarrow y \in \Omega$, and $\left\{y_{n}\right\} \rightarrow \delta$. Let $\Omega^{*}=\Omega_{L}^{*}$ be the space obtained from $\bar{\Omega}=(\partial \Omega) \cup \Omega$ by adding all $\rho$-Cauchy sequences $\left\{y_{n}\right\} \rightarrow \delta$. By virtue of the Harnack principle, $\Omega^{*}$ is compact, and for this reason, $\Omega^{*}=\Omega_{L}^{*}$ is referred to as the Martin compactification of $\Omega$ with respect to $L$. We call $\beta=\beta_{L}=\Omega^{*}-\bar{\Omega}$ the Martin ideal boundary of $\Omega$. The identity of $\bar{\Omega}$ onto $\bar{\Omega}$ is extended to a continuous map of $\bar{\Omega} \cup \beta$ onto $\bar{\Omega} \cup \delta$.

The Martin kernel $K_{s}(x, y)$ can be extended continuously to $\bar{\Omega} \times$ $(\Omega \cup \beta)$. Let $y \in \Omega$. From (34) it follows that

$$
\int_{\partial \Omega} \frac{\partial K_{\Omega}(x, y)}{\partial n_{x}} d S_{x}=1
$$

Therefore if $y^{*} \in \beta$, then

$$
\sigma\left(K_{\Omega}\left(\cdot, y^{*}\right)\right)=\int_{\partial \Omega} \frac{\partial K_{\Omega}\left(x, y^{*}\right)}{\partial n_{x}} d S_{x}=\lim _{y \rightarrow y^{*}} \int_{\partial \Omega} \frac{\partial K_{\Omega}(x, y)}{\partial n_{x}} d S_{x}=1
$$


and thus (cf. no. 2)

$$
\mathscr{K}(\Omega ; L) \equiv\left\{K_{\Omega}\left(\cdot, y^{*}\right) ; y^{*} \in \beta\right\} \subset \mathscr{P}_{1}(\Omega ; L) .
$$

11. We denote by $\overline{c o}\{\mathscr{K}(\Omega ; L)\}$ the closed convex hull of the set $\mathscr{K}(\Omega ; L)$ in $\mathscr{E}(\Omega ; L)$. We shall show that

$$
\overline{c o}\{\mathscr{K}(\Omega ; L)\}=\mathscr{P}_{1}(\Omega ; L) .
$$

For this purpose it is sufficient to show the existence of a unit regular Borel measure $\nu$ on $\beta$ for any fixed $u \in \mathscr{P}_{1}(\Omega ; L)$ such that

$$
u(x)=\int_{\beta} K_{\Omega}\left(x, y^{*}\right) d \nu\left(y^{*}\right) .
$$

Let $N$ and $N_{1}$ be in $\mathscr{N}(M)$ with $M-\Omega \subset N \subset \bar{N} \subset N_{1}$ and consider $v_{1}=$ $D\left(N_{1}-\bar{N} ; \partial N\right) u$ and $v=D(\Omega-\bar{N} ; \partial N) u$. By the Green formula

$$
u(x)=\int_{\partial N}\left(G_{c_{c}}(x, y) \frac{\partial u(y)}{\partial n_{y}}-u(y) \frac{\partial G_{\Omega}(x, y)}{\partial n_{y}}\right) d S_{y}
$$

for $x \in N \cap \Omega$, where $\partial / \partial n$ denotes the outer normal derivative with respect to the region $N \cap \Omega$. Again by the Green formula

$$
0=\int_{\partial N}\left(G_{N_{1} \cap \varsigma_{c}}(x, y) \frac{\partial v_{1}(y)}{\partial n_{y}}-v_{1}(y) \frac{\partial G_{N_{1} \cap \varsigma_{c}}(x, y)}{\partial n_{y}}\right) d S_{y} .
$$

On letting $N_{1} \rightarrow M$ we obtain

$$
0=\int_{\partial N}\left(G_{\lrcorner c}(x, y) \frac{\partial v(y)}{\partial n_{y}}-u(y) \frac{\partial G_{\Omega}(x, y)}{\partial n_{y}}\right) d S_{y} .
$$

Since $u-v_{1} \geqq 0$ on $N_{1}-N$ and $u-v_{1}=0$ on $\partial N, v_{1} \rightarrow v$ implies that $u-v \in \mathscr{P}(\Omega-\bar{N} ; L)$. A fortiori

$$
\nu_{N}(y)=e_{\Omega}(y) \frac{\partial}{\partial n_{y}}(u(y)-v(y)) \geqq 0
$$

on $\partial N$. Therefore the subtraction of (41) from (40) gives

$$
u(x)=\int_{\partial N} K_{\Omega}(x, y) d \nu_{N}(y)
$$

where $d \nu_{N}(y)=\nu_{N}(y) d S_{y}$ on $\partial N$. Observe that

$$
\int_{\partial N} d \nu_{N}(y)=\sigma(u)=\int_{\partial N}\left\{\int_{\partial \Omega} \frac{\partial}{\partial n_{y}} K_{\Omega}(x, y) d S_{x}\right\} d \nu_{N}(y)=1 .
$$

Hence there exists a subset $\left\{N_{m}\right\} \subset \mathscr{N}(M)$ such that $d \nu_{m}=d \nu_{N_{m}}$ converges vaguely to a unit regular Borel measure $d \nu$ on $\Omega^{*}$. Clearly $d \nu$ is a measure on $\beta$ and (43) implies (39). 
12. Since $\beta \subset \Omega^{*}$ is homeomorphic to $\mathscr{K}(\Omega ; L) \subset \mathscr{E}(\Omega ; L)$ by the natural correspondence $y^{*} \rightarrow K_{\Omega}\left(\cdot, y^{*}\right), \mathscr{K}(\Omega ; L)$ is compact and hence closed. By the Milman theorem (cf. e.g. Dunford-Schwartz [1]), (38) assures that Ex. $\mathscr{P}_{1}(\Omega ; L)$ is contained in the closure of $\mathscr{K}(\Omega ; L)$ which is $\mathscr{K}(\Omega ; L)$. Therefore if we set

$$
\beta_{1}=\left\{y^{*} \in \beta ; K_{\delta}\left(\cdot, y^{*}\right) \in \operatorname{Ex} . \mathscr{P}(\Omega ; L)\right\},
$$

then we conclude that

$$
\text { Ex. } \mathscr{P}_{1}(\Omega ; L)=\left\{K_{\Omega}\left(\cdot, y^{*}\right) ; y^{*} \in \beta_{1}\right\} .
$$

Since $\psi: K_{\delta}\left(\cdot, y^{*}\right) \rightarrow y^{*}$ is a homeomorphism from $\operatorname{Ex} . \mathscr{P}_{1}(\Omega ; L)$ onto $\beta_{1}$,

$$
d \mu\left(y^{*}\right)=d \nu\left(K_{\Omega}\left(\cdot, y^{*}\right)\right)
$$

is a regular Borel measure on $\beta_{1}$ if $d \nu$ is on $\operatorname{Ex} . \mathscr{P}_{1}(\Omega ; L)$. Therefore by (9) in no. 3 we deduce the fundamental theorem of Martin: there exists a bijective correspondence $u \rightarrow \mu$ between $\mathscr{P}(\Omega ; L)$ and the set of regular Borel measures on $\beta_{1}$ such that

$$
u(x)=\int_{\beta_{1}} K\left(x, y^{*}\right) d \mu\left(y^{*}\right)
$$

for $x \in \Omega$ (cf. Itô [4], Šur [10], etc.). In particular we have

$$
\operatorname{dim}(\Omega ; L)=\# \beta_{1} \text {. }
$$

Suppose $\operatorname{dim}(\Omega ; L)=1$. By (37) and (46) we see that $\# \beta=1$. Conversely $\# \beta=1$ implies that $\# \beta_{1}=1$ which in turn implies $\operatorname{dim}(\Omega ; L)=1$. Thus

Proposition. If $\operatorname{dim}(\Omega ; L)>0$, then the Picard principle is valid if and only if the Martin ideal boundary $\beta_{L}$ consists of a single point.

\section{Riemann theorem; Proof of the main theorem.}

13. We denote by $\mathscr{B}(\Omega ; L)$ the vector space of bounded solutions of (4) on $\Omega$ with continuous boundary values on $\partial \Omega$. We say that the Riemann theorem is valid for $(\Omega ; L)$ if

$$
\lim _{x \rightarrow \delta} u(x)
$$

exists for every $u \in \mathscr{B}(\Omega ; L)$. It is clear that if the Riemann theorem is valid for $\left(\Omega_{0} ; L\right)$, then it is also valid for $(\Omega, L)$ with $\Omega \supset \bar{\Omega}_{0}$. We shall discuss the Riemann theorem for $(\Omega, L)$. So far as we can consider $\hat{L}$ we have to assume $\operatorname{dim}(\Omega, L)>0$ or equivalently $\operatorname{dim}\left(\Omega, L^{*}\right)>0$. Using the operator $D^{*}=D^{*}(\Omega ; \partial \Omega)$ in (33) we maintain

$$
\mathscr{B}(\Omega ; \hat{L})=\left\{D^{*} \varphi / e_{\Omega} ; \varphi \in C(\partial \Omega)\right\} \text {. }
$$


To prove this first observe that $u$ is a solution of $L^{*} u=0$ on an open subset of $\Omega$ if and only if $v=u / e_{2}$ is a solution of $\hat{L} v=0$. Therefore $v=D^{*} \varphi / e_{\Omega}$ is a solution of $L v=0$. If $\varphi \geqq 0$, then $D^{*}(N \cap \Omega ; \partial \Omega) \varphi \leqq k e_{\Omega}$ with $k=\max _{\partial .2} \varphi$ (cf. no. 9) and a fortiori $D^{*} \varphi / e_{\Omega} \leqq k$, i.e. $D^{*} \varphi / e_{\Omega} \in$ $\mathscr{B}(\Omega ; L)$. The same is true for general $\varphi$ by considering $\varphi=\varphi^{+}-\varphi^{-}$ with $\varphi^{ \pm}=\max ( \pm \varnothing, 0)$. Conversely, let $v \in \mathscr{B}(\Omega ; \hat{L})$. Take an $N \in \mathscr{N}(M)$ with $M-\Omega \subset N$ and let $w_{N}$ be a solution of (7) on $N \cap \Omega$ with boundary values 1 on $\partial \Omega$ and 0 on $\partial N$. Then $e_{\Omega} w_{N}=D^{*}(N \cap \Omega ; \partial \Omega) 1$. Thus

$$
e_{\Omega} \lim _{N \rightarrow M} w_{N}=\lim _{N \rightarrow M} D^{*}(N \cap \Omega ; \partial \Omega) 1=e_{\Omega},
$$

i.e. $\lim _{N \rightarrow M} w_{N}=1$. If $v \mid \partial \Omega=0$ and $k=\sup _{\Omega}|v|$, then

$$
|v| \leqq k\left(1-w_{N}\right)
$$

on $N \cap \Omega$. On letting $N \rightarrow M$ we conclude that $v \equiv 0$. For general $v$, let

$$
v_{0}=v-D^{*} v / e_{\Omega} \text {. }
$$

Then $v_{0} \in \mathscr{B}(\Omega ; \hat{L})$ and $v_{0} \mid \partial \Omega=0$. Therefore $v_{0} \equiv 0$ on $\Omega$, i.e. $v=D^{*} v / e_{\Omega}$ and we deduce (47).

14. Take any $v \in \mathscr{B}(\Omega, \hat{L})$. By $(47)$ we have $e_{\Omega} v=D^{*}(\Omega ; \partial \Omega) v$. In view of (33) we have

$$
e_{\Omega}(y) v(y)=\int_{\partial \Omega} \frac{\partial G_{\Omega}(x, y)}{\partial n_{x}} v(x) d S_{x} .
$$

A fortiori, using the Martin kernel (35), we deduce the following representation

$$
v(y)=\int_{\partial \Omega} \frac{\partial K_{s}(x, y)}{\partial n_{x}} v(x) d S_{x} .
$$

Clearly the right hand side of the above is continuous on $\Omega \cup \beta_{L}$ and so is $v$. Since $v$ is continuous on $\bar{\Omega}$, we conclude that $v$ is continuous on $\Omega_{L}^{*}$ :

$$
\mathscr{B}(\Omega ; \hat{L}) \subset C\left(\Omega_{L}^{*}\right) \text {. }
$$

In particular, $\mathscr{B}(\Omega ; \hat{L}) \mid \beta_{L} \subset C\left(\beta_{L}\right)$. We maintain

Proposition. The family $\mathscr{B}(\Omega ; \hat{L}) \mid \beta_{L}$ separates points in $\beta_{L}$, i.e. for any pair $\left(y_{1}^{*}, y_{2}^{*}\right)$ of distinct points in $\beta_{L}$ there exists $a v \in \mathscr{B}(\Omega ; \hat{L})$ such that $v\left(y_{1}^{*}\right) \neq v\left(y_{2}^{*}\right)$.

Observe that, by $(48), v\left(y_{j}^{*}\right)=\int_{\partial \Omega}\left(\partial K_{\Omega}\left(x, y_{j}^{*}\right) / \partial n_{x}\right) v(x) d S_{x}(j=1,2)$ for every $v \in \mathscr{B}(\Omega ; \hat{L})$. If the assertion were not true, then 


$$
\int_{\partial \Omega} \frac{\partial k(x)}{\partial n_{x}} \varphi(x) d S_{x}=0
$$

for every $\varphi \in C(\partial \Omega)$, where $k(x)=K_{\Omega}\left(x, y_{1}^{*}\right)-K_{\delta}\left(x, y_{2}^{*}\right)$. Therefore $\partial k(x) / \partial n_{x}=0$ on $\partial \Omega$. We thus have

$$
L k(x)=0(x \in \Omega), \quad k(x)=\frac{\partial k(x)}{\partial n_{x}}=0(x \in \partial \Omega) .
$$

This implies that $k(x) \equiv 0$ on $\Omega$, i.e. $y_{1}^{*}=y_{2}^{*}$, a contradiction.

15. Proof of THE Theorem IN Introduction. We are ready to prove the theorem stated in the introduction. Since the identity of $\Omega$ can be continuously extended to a map of $\Omega \cup \beta$ onto $\Omega \cup \delta$ with $\beta$ lying over $\delta$,

$$
\limsup _{x \rightarrow \delta} u(x)=\max u\left(\beta_{L}\right), \quad \liminf _{x \rightarrow \delta} u(x)=\min u\left(\beta_{L}\right)
$$

for every $u \in \mathscr{B}(\Omega, \hat{L})$. In view of Proposition $14, \lim _{x \rightarrow \delta} u(x)$ exists for every $u \in \mathscr{B}(\Omega, \hat{L})$ if and only if $\beta_{L}$ consists of a single point, which is, by Proposition 12, equivalent to $\operatorname{dim}(\Omega, L)=1$. This completes the proof.

16. Suppose that the Riemann theorem is valid for $\left(\Omega_{0}, \hat{L}_{\Omega_{0}}\right)$. Let $\Omega$ be any end of $M$ with $\bar{\Omega} \subset \Omega_{0}$. By Proposition 7 and the main theorem, the Riemann theorem is valid for $\left(\Omega, \hat{L}_{\Omega}\right)$. Since $e_{\Omega_{0}} / e_{\Omega} \in \mathscr{B}\left(\Omega, \hat{L}_{\Omega}\right)$,

$$
\lim _{x \rightarrow \delta} e_{\Omega_{0}}(x) / e_{\llcorner}(x) \equiv \alpha>0
$$

exists. If $v$ is a bounded solution of $\hat{L}_{\Omega_{0}} v=0$ on a subend of $\Omega_{0}$ containing $\bar{\Omega}$, then $u=e_{\Omega_{0}} v$ is a bounded solution of $L^{*} u=0$ on $\bar{\Omega}$. Since $u / e_{\Omega}=\left(e_{\Omega_{0}} / e_{\Omega}\right) v, w=u / e_{.2} \in \mathscr{B}\left(\Omega, \hat{L}_{\Omega}\right)$ and

$$
\lim _{x \rightarrow \delta} v(x)=\left(\lim _{x \rightarrow \delta} w(x)\right) /\left(\lim _{x \rightarrow \delta}\left(e_{\Omega_{0}}(x) / e_{\Omega}(x)\right)=\alpha^{-1} \lim _{x \rightarrow \delta} w(x) .\right.
$$

Thus we see that the Riemann theorem for $(\Omega, \hat{L})$ is the property of ideal boundary $\delta$ in the following sense:

Proposition. The Riemann theorem is valid for $(\Omega, \hat{L})$ if and only if $\lim _{x \rightarrow \delta} u(x)$ exists for every bounded solution $u$ of $\hat{L} u=0$ on any subend of $\Omega$.

\section{An example.}

16. We take $B: 0<|z|<1(z=x+i y)$ as $\Omega$ with $\delta: z=0$ and consider

$$
L u(z)=\Delta u(z)+b(z) \cdot \nabla u(z)+c(z) u(z)
$$

on $\bar{B}: 0<|z| \leqq 1$ with the plane metric. First we remark that the 
mapping $L \rightarrow \hat{L}$ is not injective. For the purpose consider

$$
L_{P} u(z)=\Delta u(z)+2 \nabla \log e_{P}(z) \cdot \nabla u(z)+P(z) u(z)
$$

where $P(z) \geqq 0$ is of class $C^{1}$ on $\bar{B}$ and $e_{P}(z)$ is a bounded solution of

$$
\Delta u(z)=P(z) u(z)
$$

on $B$ with boundary values 1 on $\partial B:|z|=1$. The existence and the uniqueness of such an $e_{P}$ can be seen as follows. Let $u_{n}$ be a solution of (52) on $1 / n<|z|<1$ with boundary values 1 on $|z|=1$ and 0 on $|z|=1 / n(n \geqq 2)$. Since $u_{n}$ is increasing and $0<u_{n}<1, u=\lim _{n \rightarrow \infty} u_{n}$ exists on $B$ and is a required. If $v$ is another such solution, then $w=$ $(u-v)^{2}$ satisfies $\Delta w=2 P^{2}+2(\nabla w)^{2} \geqq 0$ and a fortiori $w$ is subharmonic on $B$ with $w=0$ on $\partial B$. By the minimum principle applied to the superharmonic function $-\varepsilon \log |z|-w(z)(\varepsilon>0)$, we conclude that $w \equiv 0$. The adjoint $L_{P}^{*}$ of $L_{P}$ is given by

$$
L_{P}^{*} u(z)=\Delta u(z)-2 \nabla \log e_{P}(z) \cdot \nabla u(z)+\left(2\left|\nabla \log e_{P}(z)\right|^{2}-P(z)\right) u(z) .
$$

By a direct calculation we see that $L_{P}^{*} e_{P}(z)=0$ on $B$. We wish to show that

$$
e_{P}=D^{*}(B ; \partial B) 1=e_{B} .
$$

Since $D^{*}\left(B_{n} ; \partial B\right) 1 \leqq e_{P}$ with $B_{n}: 1 / n<|z|<1$, we see that $w=e_{B} / e_{P}=$ $\lim _{n \rightarrow \infty} D^{*}\left(B_{n} ; \partial B\right) 1 / e_{P} \leqq 1$. Since $L_{P}^{*}\left(w e_{P}\right)=L_{P}^{*} e_{B}=0$ and $L_{P}^{*} e_{P}=0$, a direct calculation shows that $\Delta w=0$. Therefore $w$ is a bounded harmonic function on $B$ with boundary values 1 on $\partial B$. By the classical Riemann theorem, $w \equiv 1$ on $B$, i.e. (53) is valid.

17. In view of (53), we can rewrite $L_{P}$ as

$$
L_{P} u(z)=\Delta u(z)+2 \nabla \log e_{B}(z) \cdot \nabla u(z)+P(z) u(z) .
$$

Therefore the associated operator $\hat{L}_{P}$ to $L_{P}$ on $B$ is, by (7)

$$
\hat{L}_{P} u(z)=\Delta u(z) \text {. }
$$

Clearly $\hat{\Delta}$ on $B$ is $\Delta$. Thus we see that $L \rightarrow \hat{L}$ is not injective. Since the Riemann theorem is valid for $(B, \Delta)$, by our main theorem we conclude that the Picard principle is valid for $\left(B, L_{P}\right)$, i.e.

$$
\operatorname{dim}\left(B, L_{P}\right)=1
$$

for every $P(z) \geqq 0$ on $\bar{B}: 0<|z| \leqq 1$ of class $C^{1}$. Since we have a feeling that $\operatorname{dim}(B, L)=1$ can occur if $L$ is, in a sense, close to $\Delta,(55)$ is a rather unexpected phenomenon because $L_{P}$ may be viewed far distant from $\Delta$ if the singularity of $P$ at $z=0$ is 'wild'. This pathology pro- 
vably cannot occur if we require $L$ to be self-adjoint.

\section{REFERENCES}

[1] N. Dunford and L. Schwartz, Linear Operators, Part I, Interscience Publishers, 1957.

[2] K. HAYASHI, Les solutions positives de l'équation $\Delta u=P u$ sur une surface de Riemann, Ködai Math. Sem. Rep., 13 (1961), 20-24.

[ 3 ] M. Heins, Riemann surfaces of infinite genus, Ann. Math., 55 (1952), 296-317.

[4] S. ITô, Martin boundary for linear elliptic differential operators of second order in a manifold, J. Math. Soc. Japan, 16 (1964), 307-334.

[5] C. Miranda, Partial Differential Equations of Elliptic Type, Springer, 1970.

[6] M. NAKAI, Martin boundary over an isolated singularity of rotation free density, J. Math. Soc. Japan, 26 (1974), 483-507.

[7] M. Ozawa, Some classes of positive solutions of $\Delta u=P u$ on Riemann surfaces, I; II, Kōdai Math. Sem. Rep., 6 (1954); 7 (1955), 121-126; 15-20.

[8] R. Phelps, Lectures on Choquet's Theorem, Van Nostrand Mathematical Studies no. 7, 1965.

[9] B. Rodin and L. Sario, Principal Functions, D. Van Nostrand, 1967.

[10] M. Šur, The Martin boundary for a linear elliptic second order operator, Izv. Akad. Nauk SSSR, 27 (1963), 45-60 (Russian).

[11] K. Yosida, Functional Analysis, Springer, 1965.

Department of Mathematics

Nagoya Institute of TechNology

Gokiso, Showa, Nagoya 466

JAPAN 